\title{
A Deployment Scheme Based on Game Theory in 3D Heterogeneous Wireless Sensor Networks
}

\author{
Xiaoshuang Liu*, Jianfeng Li, Yali Wu and Hongjun Kang \\ Hebei University of Economics and Business, Information Technology College, Shijiazhuang 050061, China \\ ${ }^{*}$ Corresponding author
}

\begin{abstract}
As one principal element in Wireless Sensor Networks (WSNs), energy efficiency is very important in threedimensional (3D) heterogeneous wireless sensor networks (HWSNs). Thus, this paper considers energy balance problem in three-dimensional heterogeneous wireless sensor networks (3D HWSNs). Firstly, a nodes' deployment model in 3D HWSNs is proposed. With this model, energy saving is achieved by distributing heterogeneous Sensor Nodes (SNs) evenly in 3D HWSNs. Then, this paper presents one model based on Game Theory, which model the packet transmission between SNs. Finally, we recommend one application of the proposed network model, i.e., the hierarchy structure for Internet of Things (IoT).
\end{abstract}

Keywords-deployment; game theory; $3 D$ heterogeneous wireless sensor networks; IoT

\section{INTRODUCTION}

A wireless sensor network (WSN) comprises of large number of sensor nodes deployed in the interested region [1], which is occasionally to monitor the surroundings. SNs can work in difficult conditions where it is impossible and infeasible for the human, which is one of the main advantages of SNs [2]. Therefore, researchers like to talk about WSNs which has become a hot research field for years. In particular, 3D HWSNs is more worthy of our study. Heterogeneous network refers to heterogeneous node in this paper.

In most WSN applications, to get a good Quality of Service (QoS) [3-4] is every SN's desired state. It is very important for each SN in WSNs. Theoretically, scholars propose many algorithms, solutions and schemes for WSNs deployment issue. But a majority of them settle a matter in two-dimensional (2D) networks. However, 3D (three-dimensional) scenarios are fit for the model of true seabed terrain realistically and the requirements of many application areas. So it is the very meaningful to study the node deployment in 3D WSNs.

In heterogeneous wireless sensor networks (HWSNs), different types of SNs have different resources. The basic operation of HWSNs is as follows. Firstly, SNs can sense, inspect and gather the sensed data. And then, they transmit the data to the sink nodes. Hence, sensor nodes perform sensing, computing and transmitting. Now the major constraint of each node is its battery, that is, each of them has only limited energy. In addition, the main energy consumption of HWSNs is the communication module [5]. Therefore, the main aim of this paper is the SNs' energy utilization efficiently. How to deploy
SNs and which order to collect data are two important factors in 3D HWSNs. Hence, to schedule various activities of SNs is worthy of study. Every node should undergo the four states, transmitting, receiving, listening or idle state. Each SN needs to expend its energy in a different state. The overall energy cost of SNs can be minimized by reasonably dispatching SNs' energy consumption under monitoring and idle state [6]. During the process, each SN gets a different time slots, which has its own function to carry out, such as sending and receiving. This can prevent the data collision among sensor nodes. In energy constrained HWSNs, power-balance using could make network partitioning to be avoided, and prolong the network life [7-8]. The rigorous balancing scheme of sensor nodes' deployment process can achieve this energy balance.

Heretofore, there's been an outpouring of studies on the nodes deployment of HWSNs. The authors of [9] proposed a TDMA (Time Division Multiple Access) based system which uses the AP (Access Point) to directly synchronize and explicitly schedule the nodes' transmissions over TDMA time slots. There is the main disadvantage about this hierarchy. That is, the expenditure upon the operation of sensor clusters, intergroup communication and interference [10]. In [11], they suggested an efficient algorithm including tree-based data fusion and conflict-free fusion schedule. Furthermore, the authors obtained a latency's lower bound. In [12], the authors raised a data fusion protocol within IEEE 802.11, which is locally delayed bounded energy saving. Further, they also brought forward a energy-efficiency scheme that dispatched sensor activity. Based on cluster-based aggregation tree, the authors of [13] addressed the minimum latency scheduling algorithm. Meanwhile, they adopt a scheduling update scheme to apply in a dynamic-network topology.

For capacity consumption and load balancing, the authors of [14] came up with a node-deployment strategy to get around this problem. That is, the scattered density of SNs increases with the distance from Base Station (BS). In [15], they indicated that forwarding packets needs lots of SNs to shun the energy-hole problem. When some SNs have transmitted data exceeded their loads, an energy hole is formed. To solve this problem, many scholars have conducted researches, such as literature [16], [17], and [18]. In [16], the authors settle this matter to adjust the transmission range, which has shown to extend network life. In [17], Jun, L., and Hubaux, J.P showed a removal strategy of sink nodes to get equilibrium of SNs' power expenditure. In view of power expenditure and surplus 
energy, a load balancer's routing for SNs to locally determine a next relay SN is proposed in [18]. All the schemes listed above have their own advantages and disadvantages. The behavior of sensor nodes is selfish in a general way. They would conflict to one another to reach power utility efficiency and connectivity [19]. But these nodes being selfish would like conquering conflict objectives to achieve energy efficiency and topology connectivity. As we all know, to solve this problem raised above, Game Theory is a very effective method [20].

This paper takes the isostatic energy and network lifetime of 3D HWSNs into account. Through the research, we find that energy disequilibrium is produced when the data relay between SNs (Sensor Nodes) and BS (Base Station). We put forward a DSGT ( Deployment Scheme based on Game Theory ) in 3D HWSNs to balance SNs' energy expenditure and intensifying the 3D network lifetime. The following is introduced as shown below. Firstly, Section II shows a 3D network arrangement model, as well as perception, communication, and power models. This paper uses Game Theory to model the data transfer process in Section III. Then, we design a power equilibrium scheme for solving energy problem. Section IV describes one application of the suggested model in this paper, the hierarchy structure for the IoT. Finally, Section V concludes this paper with some mention about the future scope of our work.

\section{SYSTEM MODELS}

This Section shows a 3D network arrangement model, as well as perception, communication, and power models..

\section{A. 3D Network Arrangement Model}

There are 3-type SNs considered in 3D HWSNs, that is, Base Station (BS), Cluster Heads nodes (CHs) or Sink nodes, and Sensors Nodes (SNs). In this paper, we assume that BS has a power source, and its power is infinite. Then, BS can be the central role in 3D HWSNs. CHs and SNs have their own primary power, and expend the same power when sensing, receiving or transmitting. SNs' power is lower than CHs' power. As a general rule, we spilled the nodes (including $\mathrm{CHs}$ and SNs ) stochastically and equably, and make them surround BS. Once deployed, we can divide CA (Coverage Area) in 3D HWSNs into individual parts, that is, $3 \mathrm{D}$ regular hexagonal prism. Figure 1 shows this model detailedly. The length of the base of each prism is $r$, height of each prism is $h$, and $r=h$. Let's say 3D HWSNs is $N$-tier model. Then, the amount of prisms in tier- $i$ is $6 i(i=1,2, \ldots, N)$. The regulation is that $i=0$ shows 1 prism, i.e. BS at the center of the prism (named BS prism). As can be seen from Figure I, to tier- 0 , tier- 1 is the closest. Similarly, tier- $N$ is the farthermost. The following defines every prism with $C_{i}^{j}$, that is, the $j$-th prism in tier $i(i, j=1,2, \ldots,(6 i))$. About every prism, $\mathrm{CH}$ is located at the center, and SNs are distributed around $\mathrm{CH}$ uniformly, forming a cluster. Here, the power of $\mathrm{CH}$ is larger than its nearest SNs. To keep the whole network operation, there should be enough SNs in one prism.

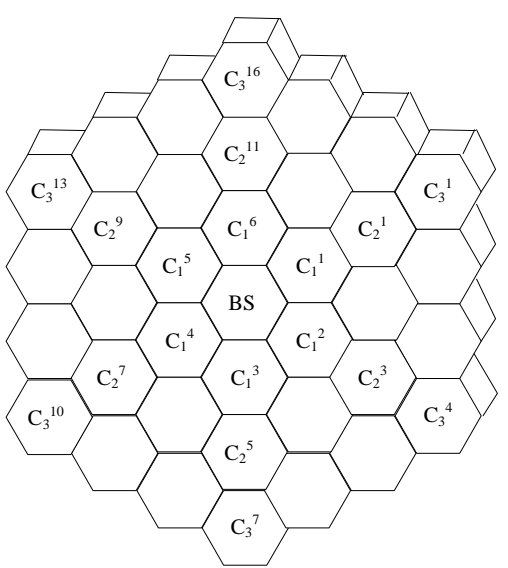

FIGURE I. 3D REGULAR HEXAGONAL PRISM MODEL

The failure node is defined as that it consumes its power lower than a certain value $\tau$. After deployment, we assume that nodes are not movable, and in an ascertainable consistency. The communication process is briefly described below. CHs gather the perceptive data by their surrounded SNs termly and conformably [21]. At the same time, the closer $\mathrm{CHs}$ also transport the received information from the farther $\mathrm{CHs}$ to BS prism. The farthest $\mathrm{CHs}$ just percept and transfer their own information. To be facilitate analysis in theory, an ideal MAC tier is defined that there is no collisions and retransmissions.

\section{B. Perception and Communication Models}

As we all know, in 1 unit volume, if all points are within the realm of 1 SN's active perception, we define the covered volume by [22]. Each node $m$ including $\mathrm{CHs}$ and SNs, has perceived and communication radius, defined as $R_{S}$ and $R_{C}$, respectively. In other words, as shown in Figure II, the perception and communication Models are spheres in 3D HWSNs, i.e., a sphere of radius $R_{S}$ or $R_{C}$ centered on $m$.

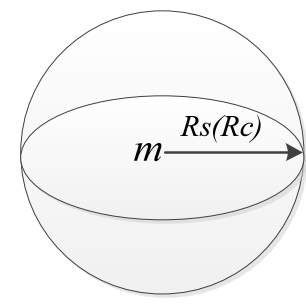

FIGURE II. 3D PERCEPTION AND COMMUNICATION MODELS

Assume that $s(m)$ is the perception space of node $m$, $c(m)$ is the communication space, and $C(M)$ is the covering space ( $M$ is on behalf of a set of SNs). Hence, $C(M)=\bigcup_{\forall m \in M} s(m)$. Here, we define that 3D HWSNs is interconnected when with 1 hop or $h$ hops any 2 nodes actively talk to one another. If two nodes in the 3D network are $X\left(x_{1}, y_{1}, z_{1}\right)$ and $Y\left(x_{2}, y_{2}, z_{2}\right)$, respectively, then the Euclidean distance $D$ between them meet 


$$
\begin{aligned}
D & =|X-Y| \\
& =\sqrt{\left(x_{1}-x_{2}\right)^{2}+\left(y_{1}-y_{2}\right)^{2}+\left(z_{1}-z_{2}\right)^{2}}
\end{aligned}
$$

$$
k_{m}=\frac{\bar{T}}{T_{m}} .
$$

It is assumed that two nodes $X$ and $Y$ can directly communicate if $D$ meet the following condition

$$
D \leq R_{C}
$$

This paper adopts the transport protocol introduced in [21], which is the communication between $\mathrm{CHs}$ and BS.

\section{Energy Model}

In one prism $C_{i}^{j}, C_{i}^{j}=\left\{c_{i}^{j}, v_{1}, v_{2}, \ldots, v_{n}\right\}$ remarks all the active SNs. Its number of nodes in this prism $C_{i}^{j}$ is $n+1 . e_{t}$ denotes 1 SN's power consumption on 1 packet transmission, and $d$ marks transmitting range. Then,

$$
e_{t}(d)=k d^{o}+e
$$

where $e$ is literal that is on behalf of all the power required for transmitting 1 packet. In addition, $o$ represents the exponent of path losses, and $k$ means the coefficient of path losses in this paper, respectively.

Assume that $e_{m}$ is the average power consumed by SN $m$ transmitting 1 data. Power expended in sensing a packet is $e_{s}$. Then,

$$
e_{m}=e_{s}+e_{t} \text {. }
$$

Let $B_{m}$ be SN $m$ 's cell capacity, and $T_{m}$ be SN $m$ 's lifetime. Therefore,

$$
T_{m}=\frac{B_{m}}{e_{m}}
$$

Hence, $\bar{T}$ denotes the average lifetime of all SNs, and

$$
\bar{T}=\frac{1}{\left|C_{l}^{j}\right|} \sum_{m \in C_{l}^{j}} T_{m},
$$

where $C_{l}^{j} \in C_{i}^{j}$, and $|\bullet|$ denotes the number of one set. $\left|C_{l}^{j}\right|$ represents the amount of SNs to participate in the packet transfer process.

We exclude these SNs if they run out of energy in $C_{i}^{j}$. Let $k_{m}$ be the RLC (Relative Life Coefficient), i.e., the ratio of the average lifetime $\bar{T}$ and SN $m$ 's lifetime $T_{m}$,

\section{ENERgy BALANCING MECHANISM}

We discuss energy balancing strategies of 3D Regular hexagonal prism model in 3D HWSNs in this segment. First, we recommend the packet transfer process based on Game Theory in Section III. Figure III shows 3 main periods in this process, that is, sensing period, transmitting period and receiving period.

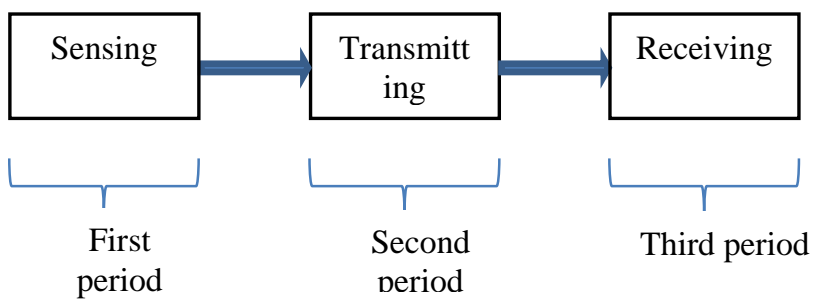

FIGURE III. THE DATA TRANSMISSION PROCESS

We assume $F_{m}$ is the reaction function in this paper. All the SNs want their own $F_{m}$ maximization in Game Theroy, but they must take other SNs' strategies into account. We describe the above process as a Game, denoted as $G\left(C_{l}^{j}, P, F\right)$. In prism $C_{l}^{j}$, Node $m$ has its own tactics $p_{m}$, and $F_{m}$ is its settlement. As a result, we can get a Nash equilibrium if all the nodes couldn't improve their performance criterion through adjusting their strategies unilaterally. $p^{\prime}=\left(p_{1}, p_{2}, \ldots, p_{\left|c_{l}^{j}\right|}\right)$ is a Nash equilibrium point, when

$$
F_{m}\left(p^{\prime}\right) \geq F_{m}\left(p_{-m}^{\prime}, p_{m}\right), \forall m \in C_{l}^{j}, 0 \leq p_{m} \leq 1
$$

The above analysis shows that the appropriate adjustments of relevant parameters in this paper meet conclusions, in order to realize energy balancing. The equilibrium strategy is computed by $\mathrm{CH}$ due to the physical constraints of SNs in one prism. When this process finished, all SNs involved in this cluster will get the computation. In a cluster, a status chart about all SNs is kept in CH. To calculized a equilibrium set, the status chart should include one SN's association attributes, liking energy consumption, alive or dead.

\section{APPLICATION}

Section IV gives one application of the suggested models above, that is, a hierarchy structure of IoT. It also shows the traffic process of 3D regular hexagonal prism model. In Figure IV, RNs (or CHs) are made up of RFID readers, advanced processing and communication units, and their role is to fuse the packet collected by SNs and relay data to a BS. Several BSs interconnect to form a network which further uploads packet to the Internet. 


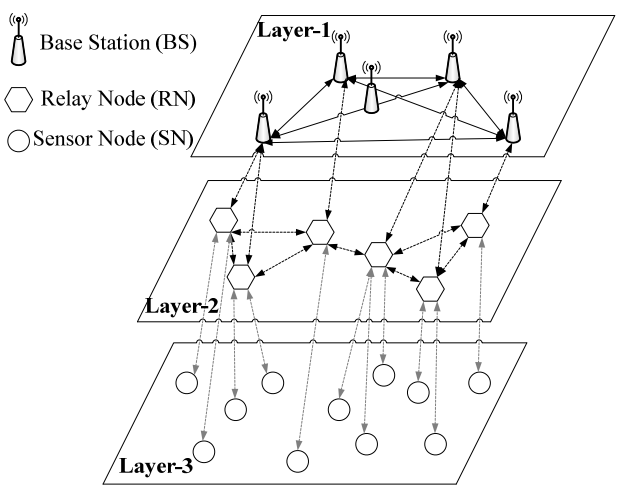

FIGURE IV. SYSTEM STRUCTURE FOR IOT DEPLOYMENT

\section{CONCLUSION}

In this paper, to solve the energy-hole problem in 3D HWSNs, we study energy balance tactics which can enhance the network lifetime effectively. This paper lists 3D Network arrangement model, perception, communication, and power models.in detail. The data transfer process can be modeled as a Game to achieve energy fairness. Finally, one application is given to model the communication process of the suggested scheme, a hierarchy structure of IoT. Studying the other energy consumption models with the consideration of coverage, connectivity and energy efficiency in the IoT is our future research.

\section{ACKNOWLEDGMENT}

This work is supported in part by Research project of Hebei University of Economics and Business (No. 2016KYQ02) and (No. 2016KYQ03), Educational project of Hebei University of Economics and Business (No. 2018JYQ09) and (No. 2018JYQ10), National Natural Science Foundation of China (61801165), Natural Science Foundation of Hebei Province, China (F2017207006), and the Science and Technology Fund of Hebei Educational Committee for Youths (No. QN2016178).

\section{REFERENCES}

[1] Fan G J, Jin S Y. Coverage problem in wireless sensor network: A survey[J]. Journal of networks, 2010, 5(9): 1033..

[2] Mainwaring A, Culler D, Polastre J, Szewczyk, R., and Anderson, J, "Wireless sensor networks for habitat monitoring," Proceedings of the 1st ACM international workshop on Wireless sensor networks and applications. ACM, 2002: 88-97.

[3] S. Meguerdichian, F. Koushanfar, M. Potkonjak, M. B. Srivastava, "Coverage Problems in Wireless Ad-hoc Sensor Networks," IEEE INFOCOM’01, April 2001.

[4] K. Kar, S. Banerjee, "Node Placement for Connected Coverage in Sensor Networks,” WiOpt'03: Modeling and Optimization in Mobile, Ad Hoc and Wireless Networks, March 2003.

[5] Halgamuge M N, Mendis P, Aye L, et al., "Energy Optimized Wireless Sensor Network for Monitoring inside Buildings: Theoretical Model and Experimental Analysis,” Progress In Electromagnetics Research M, 2014, 37: 11-20.

[6] Shu, F., M. N. Halgamuge, and W. Chen, "Building automation systems using wireless sensor networks: Radio characteristics and energy efficient communication protocols,” EJSE Special Issue: Sensor
Network on Building Monitoring: From Theory to Real Application, 6673, 2009.

[7] S. Olariu, and I. Stojmenovic, "Design Guidelines for Maximizing Lifetime and Avoiding Energy Holes in Sensor Networks with Uniform Distribution and Uniform Reporting,” Presented at the INFOCOM 2006. 25th IEEE International Conference on Computer Communications. Proceedings, April 2006, pp. 1-12.

[8] R. Fengyuan, Z. Jiao, H. Tao, L. Chuang, and S. K. D. Ren, "EBRP: Energy-Balanced Routing Protocol for Data Gathering in Wireless Sensor Networks,” Parallel and Distributed Systems, IEEE Transactions on, vol. 22, 12, pp. 2108-2125, 2011.

[9] Sinem Coleri, Anuj Puri, Pravin Varaiya, "Power Efficient System for Sensor Networks", proceedings of 8th IEEE International Conference on Computers and Communication, July 2003.

[10] Shrivastava P, Pokle S B. "Energy Efficient Scheduling Strategy for Data Collection in Wireless Sensor Networks," Electronic Systems, Signal Processing and Computing Technologies (ICESC), 2014 International Conference on. IEEE, 2014: 170-173.

[11] Xiang Yang Li, Xiao Hua Xu, Shi Guang Wang, Shao Jie Tang, Guojun Dait, J i Zhong Zhao, Yong Qi, "Efficient Data Aggregation in Multi hop Wireless Sensor Networks under Physical Interference Model”, IEEE,2009.

[12] Xu Li, Shuo Yan, Chendong Xu, Amiya Nayak,Ivan Stojmenovic, "Localized Delay Bounded and Energy Efficient Data Aggregation in Wireless Sensor Networks”, International Conference on Wireless Communication and Mobile Computing, 2011.

[13] Yingshu Li, Longjiang Guo, Sushil K.Prasad, “An Energy Efficient Distributed Algorithm for Minimum Latency Aggregation Scheduling in Wireless Sensor Networks,” Proceedings of 30th IEEE International conference, June 2010.

[14] Yick, J., Mukherjee, B., and Ghosal, D., "Wireless sensor network survey,” Computer networks, 2008, 52, (12), pp. 2292-2330.

[15] Lloyd E L, Xue G. Relay node placement in wireless sensor networks[J]. IEEE Transactions on Computers, 2006, 56(1): 134-138.

[16] Yang D, Misra S, Fang X, et al. Two-tiered constrained relay node placement in wireless sensor networks: Efficient approximations[C]//2010 7th Annual IEEE Communications Society Conference on Sensor, Mesh and Ad Hoc Communications and Networks (SECON). IEEE, 2010: 1-9..

[17] Luo, Jun, and Jean-Pierre Hubaux. "Joint sink mobility and routing to maximize the lifetime of wireless sensor networks: the case of constrained mobility." IEEE/ACM Transactions on Networking (TON) 18.3 (2010): 871-884.

[18] Ammari, Habib M., and Sajal K. Das. "Promoting heterogeneity, mobility, and energy-aware voronoi diagram in wireless sensor networks." IEEE Transactions on Parallel and Distributed Systems 19.7 (2008): 995-1008.

[19] Abbasi M J, Bin Abd Latiff M S, Chizari H, et al., "Game Theory Based Construction Efficient Topology in Wireless Sensor Networks," Mathematical Problems in Engineering, 2015.

[20] M. Abbasi, M. S. Bin Abd Latiff, and H. Chizari, "An overview of distributed energy-efficient topology control for wireless Ad Hoc networks,” Mathematical Problems in Engineering, vol. 2013, Article ID839486, 16 pages, 2013.

[21] Halder, Subir, and Sipra Das Bit. "Enhancement of wireless sensor network lifetime by deploying heterogeneous nodes." Journal of Network and Computer Applications 38 (2014): 106-124.

[22] Halder, Subir, Amrita Ghosal, and Sipra Das Bit. "A pre-determined node deployment strategy to prolong network lifetime in wireless sensor network." Computer Communications 34.11 (2011): 1294-1306. 